\title{
Genomic Variations in SARS-CoV-2 Strains at the Target Sequences of Nucleic Acid Amplification Tests
}

\section{Canhui Cao}

National Medical Center for Major Public Health Events, Tongji Hospital, Tongji Medical College, Huazhong University of Science and Technology

\section{Ruidi Yu}

National Medical Center for Major Public Health Events, Tongji Hospital, Tongji Medical College, Huazhong University of Science and Technology, Wuhan, People's Republic of China

\section{Shaoqing Zeng}

National Medical Center for Major Public Health Events, Tongji Hospital, Tongji Medical College, Huazhong University of Science and Technology

\section{Dan Liu}

National Medical Center for Major Public Health Events, Tongji Hospital, Tongji Medical College, Huazhong University of Science and Technology

\section{Wenjian Gong}

National Medical Center for Major Public Health Events, Tongji Hospital, Tongji Medical College, Huazhong University of Science and Technology

\section{Ruyuan Li}

National Medical Center for Major Public Health Events, Tongji Hospital, Tongji Medical College, Huazhong University of Science and Technology

\section{Siyuan Wang}

National Medical Center for Major Public Health Events, Tongji Hospital, Tongji Medical College, Huazhong University of Science and Technology

\section{Yuan Yuan}

National Medical Center for Major Public Health Events, Tongji Hospital, Tongji Medical College, Huazhong University of Science and Technology

\section{Jianhua Chi}

National Medical Center for Major Public Health Events, Tongji Hospital, Tongji Medical College, Huazhong University of Science and Technology

Jiahao Liu

National Medical Center for Major Public Health Events, Tongji Hospital, Tongji Medical College, Huazhong University of Science and Technology

\section{Yang Yu}


National Medical Center for Major Public Health Events, Tongji Hospital, Tongji Medical College, Huazhong University of Science and Technology

\section{Xiaofei Jiao}

National Medical Center for Major Public Health Events, Tongji Hospital, Tongji Medical College, Huazhong University of Science and Technology

\section{Guangyao Cai}

National Medical Center for Major Public Health Events, Tongji Hospital, Tongji Medical College, Huazhong University of Science and Technology

\section{Ning Jin}

National Medical Center for Major Public Health Events, Tongji Hospital, Tongji Medical College, Huazhong University of Science and Technology

\section{Fei Ye ( $\square$ yeyuanbei@hotmail.com )}

Department of Neurosurgery, Tongji Hospital, Tongji Medical College, Huazhong University of Science and Technology

\section{Qinglei Gao ( $\sim$ qingleigao@hotmail.com )}

National Medical Center for Major Public Health Events, Tongji Hospital, Tongji Medical College, Huazhong University of Science and Technology

\section{Research Article}

Keywords: SARS-CoV-2, nucleic acid tests, genome variations, COVID-19, primers of PCR

Posted Date: October 21st, 2020

DOI: https://doi.org/10.21203/rs.3.rs-95881/v1

License: (c) (1) This work is licensed under a Creative Commons Attribution 4.0 International License. Read Full License

Version of Record: A version of this preprint was published at Archives of Medical Science on March 21st, 2021. See the published version at https://doi.org/10.5114/aoms/133120. 


\section{Genomic Variations in SARS-CoV-2 Strains at the Target Sequences of Nucleic Acid Amplification Tests}

Canhui Cao $^{1 \text { II, }}$ Ruidi Yu ${ }^{1 \pi}$, Shaoqing Zeng ${ }^{1}$, Dan Liu ${ }^{1}$, Wenjian Gong1, Ruyuan $\mathrm{Li}^{1}$, Siyuan Wang ${ }^{1}$, Yuan Yuan ${ }^{1}$, Jianhua Chi ${ }^{1}$, Jiahao Liu ${ }^{1}$, Yang Yu ${ }^{1}$, Xiaofei Jiao $^{1}$, Guangyao Cai ${ }^{1}$, Ning Jin ${ }^{1}$, Fei Ye ${ }^{2 *}$ \& Qinglei Gao ${ }^{1^{*}}$

1 National Medical Center for Major Public Health Events, Tongji Hospital, Tongji Medical College, Huazhong University of Science and Technology, Wuhan, People's Republic of China

2 Department of Neurosurgery, Tongji Hospital, Tongji Medical College, Huazhong University of Science and Technology, Wuhan, People's Republic of China.

* Correspondence:

Qinglei Gao

qingleigao@hotmail.com

Fei Ye

yeyuanbei@hotmail.com

IThese authors contributed equally to this work. 


\begin{abstract}
Background: Nucleic acid amplification is the main method used to detect infections of severe acute respiratory syndrome coronavirus 2 (SARS-CoV-2). However, the false-negative rate of nucleic acid tests cannot be ignored.
\end{abstract}

Methods: Herein, we demonstrated genomic variations at the target sequences for the tests and the geographical distribution of the variations across countries by analyzing the whole-genome sequencing data of SARS-CoV-2 strains from the 2019 Novel Coronavirus Resource (2019nCoVR) database.

Results: Among the 21 pairs of primer sequences in regions ORF1ab, S, E, and N, the total length of primer and probe target sequences was 938bp, with 131(13.97\%) variant loci in 2415 (38.96\%) isolates. Primer targets in the $\mathrm{N}$ region contained the most variations that were distributed among the most isolates, and the E region contained the least. Single nucleotide polymorphisms were the most frequent variation, with $\mathrm{C}$ to $\mathrm{T}$ transitions being detected in the most variant loci. $\mathrm{G}$ to $\mathrm{A}$ transitions and $\mathrm{G}$ to $\mathrm{C}$ transversions were the most common and had the highest isolate density. Genomic variations at the three mutation sites N: 28881, N: 28882 , and N: 28883 were the most commonly detected, including in 608 SARS-CoV-2 strains from 33 countries, especially in the United Kingdom, Portugal, and Belgium.

Conclusions: Our work comprehensively analyzed genomic variations on the target sequences of the nucleic acid amplification tests, offering evidence to optimize primer 
and probe target sequence selection, thereby improving the performance of the SARSCoV-2 diagnostic test.

Keywords: SARS-CoV-2, nucleic acid tests, genome variations, COVID-19, primers of PCR

\section{Background}

The coronavirus disease 2019 (COVID-19) outbreak has resulted in a Public Health Emergency of International Concern since 30 January 2020, and has been classified as an ongoing pandemic since 11 March 2020 [1,2]. There have been 5,000,561 confirmed infected cases and 328,172 deaths by as of May 21, 2020, involving 229 countries and territories worldwide [3]. COVID-19 is an infectious disease caused by the severe acute respiratory syndrome coronavirus 2 (SARS-CoV-2) virus [4], which transmits rapidly via human respiratory droplets, direct contact, and aerosol transmission [5]. The widespread popularity and rapid spread of COVID-19 poses an urgent need for fast and accurate diagnosis methods.

Currently, the diagnosis of COVID-19 relies on positive pathogenetic, epidemiological exposure history, CT imaging features, and serological characteristics [6, 7], as well as symptoms that are atypical and might be confused with influenza, common cold, and pneumonia caused by other pathogens [8-11]. Positive pathogenicity is commonly confirmed by nucleic acid testing via reverse transcription polymerase chain reaction (RT-PCR), virus isolation and sequencing, or specific antibody detection. Currently, nucleic acid testing in various bodily fluids is the primary method of diagnosing 
COVID-19 $[9,12]$. This is similar to the diagnostic approach for SARS and MERS, the two other coronavirus diseases [13-15]. Hence, the sensitivity and specificity of nucleic acid testing are crucial. Although it is highly specific, the sensitivity is not satisfactory [16]. There are various factors that may interfere with test sensitivity. The sampling days from onset of symptoms, different sites used to obtain a specimen, insufficient viral material in the specimen, laboratory error during sampling, and restrictions on sample transportation could all influence the nucleic testing and cause false-negative results [17-20]. However, few studies mention false negatives due to variations in primer target sequences.

The RT-PCR kits have been designed to detect SARS-CoV-2 genetically, involving the reverse transcription of SARS-CoV-2 RNA into complementary DNA (cDNA) strands, followed by amplification of specific regions of the cDNA with paired primers and probes [21]. Among the SARS-related viral genomes, there are three regions with conserved sequences: 1) the RdRP gene (RNA-dependent RNA polymerase gene) in the open reading frame ORF1ab region, 2) the E gene, and 3) the $\mathrm{N}$ gene [22]. Both the RdRP and E genes had high analytical sensitivity for detection, whereas the $\mathrm{N}$ gene provided poorer analytical sensitivity $[9,22]$. Thus, some assays were designed as a two-target system, where one primer set universally detected coronaviruses, and a second set only detected SARS-CoV-2 [9]. As of 21 May 2020, 315 commercialized molecular assays had been designed to detect SARS-CoV-2 genetically, worldwide, according to information provided by The Foundation for Innovative New Diagnostics, some PCR protocols had been shared [23, 24]. The sensitivity of nucleic acid detection 
kits relies on the binding of primers and probes to the virus genome [9, 25]. High variation frequency, especially the variation near the $3^{\prime}$ end, might influence the primers or probes binding to the virus genome [26]. Thus, variations in the target sequence and how these variations affect the accuracy of the tests need to be elucidated.

SARS-CoV-2 is a positive-sense single-stranded RNA virus, with a genome that has typical betacoronavirus organization: a 5' untranslated region (UTR), replicase complex (orf1ab), S (spike) gene, E (envelope) gene, M (membrane) gene, $\mathrm{N}$ (nucleocapsid) gene, 3' UTR, and several unidentified nonstructural open reading frames [27-30]. T RNA virus's mutation rate is dramatically high, and this high rate is correlated with virulence modulation and evolvability for viral adaptation [31]. It has been reported that the most frequent variations are found within open-reading frame (ORF) regions [32], however, some studies suggest that overall variation in ORF regions is low [33]. Therefore, the variation frequency in the aforementioned regions of the genome needs further study, especially in the primer and probe target sequence.

Although most primer and probe sets were designed to focus on specific and conserved sequences, there were still some variations in the target sequences of the nucleic acid test assays of SARS-CoV-2 strains. A number of studies compared the sensitivity and efficiency of different SARS-CoV-2 detection assays [34, 35]. But few people pay attention to the variations and geographic distribution that may affect the PCR performance of detection kits used on a large scale in the population. Herein, we comprehensively analyzed genomic variations in the target sequences of the nucleic 
acid amplification tests and their geographic distribution. Our results are expected to provide evidence for optimizing the selection of detection kits used on a large scale in the population, thereby improving the diagnostic accuracy of the tests.

\section{Methods}

\section{Novel Coronavirus Resource}

We used the 2019 Novel Coronavirus Resource [36, 37], constructed by the Chinese Academy of Sciences. It integrates genomic and proteomic sequences as well as their metadata from the Global Initiative on Sharing All Influenza Data, National Center for Biotechnology Information, China National GeneBank, National Microbiology Data Center, and China National Center for Bioinformation/National Genomics Data Center. The 2019 nCoVR offers visualization functionalities for genomic variation analysis results based on all SARS-CoV-2 strains collected.

\section{Source of primers and probes used in nucleic acid detection kits.}

The WHO offered molecular assay protocols for SARS-CoV-2 that have been shared (WHO, 2020), including seven institutes: the Chinese Center for Disease Control and Prevention (China CDC); Institute Pasteur, Paris, France (IP France); Centers for

Disease Control and Prevention, the United States (US CDC); National Institute of Infectious Diseases, Japan (NIID Japan); Charité, Germany; School of Public Health, Hong Kong University (HKU); and the National Institute of Health, Thailand (NIH 
Thailand). Meanwhile, 21 pairs of primers and probes from the above protocols were mapped to the reference genome.

\section{Mapping of genomic variations}

The sequences of primers and probes were mapped to the SARS-CoV-2 isolate WuhanHu-1, complete genome (NCBI Reference Sequence: NC_045512.2) [36]. The positions of the primers and probes used in the nucleic acid testing kits were identified. Then, the sequences of the primers and probes were compared with the variant loci offered by 2019 nCoVR, with the variant loci and corresponding isolates downloaded from the database.

\section{Variation curve}

Virus isolates with variations at each site divided by country at each time point were counted. The curve shows the trends of isolates from different countries over time at some variant loci.

\section{Variation density and isolate density calculation}

In ORF1ab, S, E, and R regions of the SARS-CoV-2 genome, the length of primers and probes, and the number of variant loci covered by the primers and probes in each region were calculated first. The variation density of a region was the number of variant loci divided by the length of primers and probes. In addition, the number of isolates presenting variations in the primer and probe target sequence was calculated. Isolate density was the number of isolates divided by the length of the target sequence. 


\section{The variance of time and regional variations}

First the frequency of population occurrence at each variant loci over time or country was identified. Time variance was the variance based on the frequency of population occurrence at each time point, to assess the dispersion of changes at that site. Taking the country as a unit, we calculated regional variance based on the frequency of population occurrence in each country, to evaluate the dispersion of variations at that location.

\section{Statistical analysis}

The majority of statistical analyses were performed using SPSS software package version 22. The chi-square test was used to compare the differences in counting data between groups. Variance was used to evaluate the degree of dispersion as described above. A two-sided $\mathrm{P}$ value less than 0.005 was considered statistically significant.

\section{Results}

\section{Variation landscape of primer and probe target sequences in the SARS-CoV-2}

\section{strains}

Twenty-one pairs of primers and probes obtained from seven institutes worldwide were analyzed. The primers and probes were mainly focused on the replicase complex (ORF1ab), spike (S), envelope (E), and nucleocapsid (N) regions. The total length of primer and probe target sequence was $938 \mathrm{bp}$, with 131 (variation density=13.97\%) variant loci. In addition, 2415 (38.96\%, isolate density=2.57 per locus) of the 6198 
SARS-CoV-2 strains from the 2019 Novel Coronavirus Resource (2019nCoVR) database had variations in the target sequence (Table 1).

Among the four regions, most primers and probes were located in the ORF1ab region. They covered 484 - 18909, including 42 variant loci and 271 SARS-CoV-2 strains, with variations at these sites. The most common variant loci in the ORF1ab region were 514 ( 87 virus strains, $32.10 \%$ of the 271 strains), followed by 13402, and 490 (Fig 1A). As for the S region, there were only three pairs of primers covered from 24354 to 24900. There were 12 variant loci and 34 SARS-CoV-2 strains, with 24368 (14 virus strains, $41.18 \%$ of the 34 strains) being the most common variant loci (Fig 1B). Interestingly, there were two pairs of primers and probes provided by two different institutes located in the E region, and the two pairs were the same. It covered 26269 to 26381 . Barely five variant loci and eight SARS-CoV-2 strains were discovered. The most common variant loci were located at 26340 , with four virus strains that had variation at this site, occupying $50 \%$ of the eight virus strains (Fig 1C). Although the $\mathrm{N}$ region only had seven pairs of primers and probes covered from 28287 to 29282 , it had the most variations (72 loci), and involved virus strains (2102, 5.8 per locus, $33.91 \%$ of the 6198 SARS-CoV-2 virus strains from the database) among the four regions. The most common variant loci in the $\mathrm{N}$ region were 28881 with 609 virus strains (28.97\% of the 2102 virus strains), 28882 with 608 virus strains (28.92\%), and 28883 with 608 virus strains $(28.92 \%)$. These three variant loci had the most related isolates among the four regions (Fig. 1D). 
The length of primer and probe target sequence in the ORF1ab region was $415 \mathrm{bp}$, which is the longest among the four regions, with 42 variant loci (variation density = 0.10). The $\mathrm{N}$ region, the most mutative region, had the most variant loci (72 loci) and the highest variation density (0.20), with a $365 \mathrm{bp}$ target sequence length. The primer or probe target sequences in the S region were only 84 bp in length. However, only 12 variant loci were identified with high variation density $(0.14)$. The target sequence of the E region was the shortest (74 bp) and the least mutative, with the least variant loc (5 loci) and the lowest variation density $(0.07, \mathrm{p}=0.024)$ (Figs. 1E and $1 \mathrm{~F})$.

Among these four regions, there were 2415 (isolate density=2.57 per locus) virus strains with variant loci among primer or probe target sequences. In ORF1ab, 271 (isolate density $=0.65$ per locus) isolates had variant loci involved in the primers or probes, and 34 isolates (isolate density $=0.40$ per locus) in the $\mathrm{S}$ region. Significantly, the least isolates and isolate density were demonstrated in the E region, with 8 strains and 0.11 isolates per locus. Conversely, the $\mathrm{N}$ region had the most isolates and the highest density, with 2102 isolates and 5.78 isolates per locus (Fig. 1G).

\section{Variation analysis of SARS-CoV-2 strains in the target sequence of nucleic acid amplification tests.}

To further illustrate the variations, we investigated the specific variation types. Among the variant loci covered by paired primers and probes, the most common variations were single nucleotide polymorphisms (SNPs), accounting for $96 \%$ of all variations observed. Only $3 \%$ of the variations were deletions, and $1 \%$ of the variations had SNPs and 
deletions (Fig 2A). Regarding the involved SNPs, 58\% were transitions and 35\% were transversions. A total of $37 \%$ of SNP variant loci were $\mathrm{C}$ to $\mathrm{T}$ transitions, the most common type of SNP, followed by $\mathrm{G}$ to $\mathrm{T}$ transversions and $\mathrm{T}$ to $\mathrm{C}$ transitions (Fig 2B).

In the 6198 SARS-CoV-2 virus strains, the $\mathrm{G}$ to A transition involved the most isolates (1218) with the highest isolate density (121.8 per locus) in all types of SNPs, followed by $\mathrm{G}$ to $\mathrm{C}$ transversion and $\mathrm{T}$ to $\mathrm{C}$ transition. $\mathrm{T}$ to $\mathrm{G}$ transversion and $\mathrm{C}$ to $\mathrm{T}$ transitions showed the opposite trends. The former were found in fewer virus strains, but at a higher density (53 strains, 17.67 strains per site), while the latter were found in more virus strains, but at a lower density (172 strains, 3.25 strains per site) (Fig 2C).

The $\mathrm{C}$ to $\mathrm{T}$ transition was the most common SNP type, accounting for $42 \%$ of the SNPs in the ORF1ab region, $38 \%$ in the $\mathrm{S}$ region, $60 \%$ in the $\mathrm{E}$ region, and $39 \%$ in the $\mathrm{N}$ region. The ORF1ab region contained 12 SNPs. Following the $\mathrm{C}$ to $\mathrm{T}$ transition, the common SNPs were $\mathrm{G}$ to $\mathrm{T}$ transversion, $\mathrm{T}$ to $\mathrm{C}$ transition, and $\mathrm{A}$ to $\mathrm{G}$ transition. There were eight SNP types in the S region. In addition to the $\mathrm{C}$ to $\mathrm{T}$ transition and $\mathrm{A}$ to $\mathrm{G}$ transition (15\%), the other six types uniformly accounted for 7\%-8\% separately. The least SNP types were in the E region, with only three types of SNPs; C to T transition, $\mathrm{T}$ to A transversion (20\%), and $\mathrm{T}$ to $\mathrm{C}$ transition (20\%). In the $\mathrm{N}$ region, there were 16 types of SNPs, the most abundant. Besides the $\mathrm{C}$ to $\mathrm{T}$ transition, the most common type of SNPs were $\mathrm{G}$ to $\mathrm{T}$ transversion and $\mathrm{T}$ to $\mathrm{C}$ transition (Fig 2D).

Variation analysis of $\mathrm{N}: 28881, \mathrm{~N}: \mathbf{2 8 8 8 2}$, and $\mathrm{N}: 28883$ loci. 
To investigate the variations at the most common variant loci, we analyzed the occurrence of related primers, isolates, and variations over time and within different geographical regions. The variant loci that involved the most isolates among the primers or probes covered region were $\mathrm{N}: 28881(609), \mathrm{N}: 28882$ (608), and N:28883 (608). The primers covering these three variant loci were, F: GGGGAACTTCTCCTGCTAGAAT, and R: CAGACATTTTGCTCTCAAGCTG, recommended by China CDC. Besides 28881-28883, some variant loci had many corresponding mutant virus strains, such as 28887, 28896, and 28969 (Fig 3A). The virus strains with variations at 28881, 28882, and 28883 had 608 overlapping strains. Only one strain was unique, shown at 28881 (Fig 3B). The variation tendencies of the isolates involved in the above three variant loci were almost the same. At the end of February, major mutant isolates were obtained from Switzerland, at the end of March and the beginning of April, the United Kingdom (UK) provided a large portion of the mutant isolates, followed by Belgium and Portugal. The time variances of the tree sites were the same (Fig 3C). In detail, the unique strain, at 28881, came from Australia, with 45 isolates at this site. In other countries, except Australia, the trend was the same at 28881, 28882, and 28883. Most isolates came from the UK, with 140 mutant isolates at these three sites separately. The following were from Belgium, Netherlands, Portugal, and Iceland. The variation frequencies of the three sites were also the same, showing different geographical preferences with isolate distribution. The highest variation frequency was from Mexico (1.00), whose mutant isolates were extremely limited. 
Greece and Portugal also had high variation frequencies at the three sites. The regional variances of the three sites equaled 0.0424 (Fig 3D).

\section{Isolate analysis of common variant loci.}

Geographical distributions of several other common variant loci that involved many isolates were also demonstrated. At ORF1ab:490, there were $21(60.00 \%)$ mutant isolates from Australia, and $11(31.43 \%)$ from the United States. The highest variation frequencies were in New Zealand and Georgia $(0.125$, regional variance $=0.0007)($ Fig 4A). And the forward primer of the first pair of primers and probes from Japan National Institute of Infectious Diseases targeted this variant locus (Fig 1A and Table 1). At ORF1ab:514, most mutant isolates were from the Netherlands (66 isolates, $75.86 \%$ ) with a high variation frequency (0.12). The highest variation frequencies were observed in Slovakia (Fig 4B). And this variant locus was targeted by the forward primer of No.3 pair of primers and probes from Japan National Institute of Infectious Diseases (Fig 1A and Table 1). Prominently, 96.08\% (49 isolates) mutant isolates at ORF1ab:13402 were from Belgium with the second-highest variation frequency. The highest variation frequency was from Denmark (0.29, regional variance=0.0020) (Fig 4C). As for $\mathrm{N}: 28311$, targeted by No.5 pair of primers and probes provided by China CDC (Fig 1D and Table 1), 20 (57.14\%), isolates were from Australia, which was the highest. However, the variation frequency of strains from Australia was not high. The highest variation frequencies occurred in Saudi Arabia, South Korea, and Malaysia (regional variance $=0.0053)($ Fig 4D). The probe of No.15 pair of primers and probes provided by 
US CDC targeted this variant locus (Fig 1D and Table 1). The distribution of mutant isolates at N:28688 was decentralized. It was targeted by the forward primer of No.17 pair of primers and probes, which was recommended by US CDC (Fig 1D and Table 1). There were $31(36.47 \%)$ isolates from Australia, 18 (21.18\%) from China, and 11 (12.94\%) from India. High variation frequencies were observed in Turkey, India, and Kuwait (regional variance=0.0273) $($ Fig 4E).

\section{Discussion}

In the present study, our fundamental purpose is to reveal the variations in the target sequence of primers and probes in COVID19 detection kits used on a large scale in the population. We compared the primer and probe sequences used to detect SARS-CoV2 infection to those exported from the 2019 nCoVR database to gain important insights into virus variations in primer and probe target regions.

By integrating 21 pairs of primer sequences in regions ORF1ab, S, E, and N from 6198 SARS-CoV-2 strains, we found 31(13.97\%) variant loci, involving 2415 (38.96\%) isolates, especially in the $\mathrm{N}$ region, which had the highest variation density and the highest isolate density. The most variant loci and the most isolates were also located in $\mathrm{N}$ region (Figs $1 \mathrm{~F}$ and $1 \mathrm{G}$ ). The high variation frequency and included isolate density implied that the $\mathrm{N}$ region might not be suitable for primer design, which might elevate the false-negative rate. Therefore, merely nucleic acid testing alone is not enough to suppose a negative case, it should be combined with other tests such as antibody 
detection [9]. In contrast, the lowest variation density and the lowest isolate density were observed in the E region. In addition, it had the least variant loci and the least isolates. Thus, the E region might be the most conserved region, suitable for primer design [26]. This is consistent with the findings of Buddhisha et al. and Corman et al. that the RdRP and E genes had high analytical sensitivity for detection, whereas the $\mathrm{N}$ gene provided poorer analytical sensitivity $[9,22,38]$. However, only two identical pairs of primers and probes were from the E region, which might cause an incomplete understanding of this region in the present study.

The variations involved in the primers and probes were mainly SNPs, which were abundant in the SARS-CoV-2 genome [39-41]. Recent research has demonstrated that SNPs in SARS-CoV-2 are capable of substantially changing their pathogenicity [41]. Importantly, a small number of SNPs in the primer target sequence might not cause primer binding failure [26]. The $\mathrm{C}$ to $\mathrm{T}$ transition was the most common type of SNP in the primer and probe target sequence. $\mathrm{N}: 28881, \mathrm{~N}: 28882$, and $\mathrm{N}: 28883$ were the most influential variant loci, involving 608 or 609 viral isolates. It would be better to avoid designing primers or probes whose target sequences contain these three sites. As only the No. 18 primer pair targeted this sequence in the 21 pairs, verification with different nucleic acid detection kits or ddPCR could elevate sensitivity [42, 43]. As viruses evolve during outbreaks, SNP in primer or probe binding regions could alter the sensitivity of PCR assays [35]. We analyzed the variations in the target sequence of primers and probes in COVID19 detection kits used on a large scale in the population and their geographic distribution. Thereby, providing a reference for optimizing the 
selection of detection kits used on a large scale in the population, improving the diagnostic accuracy of the tests. Sapoval et al. figured out that each probe/primer sequence that was available on the WHO website contained 2.529 iSNV and/or 2.477 SNPs, suggesting the potential for a drop in the sensitivity of the affected probes and primers. They also noted that the potential impact of iSNVs on primer and probe binding should not be overlooked given the possibility of iSNVs establishing as SNPs [44]. And there were already some researches about how would SNPs in SARS-CoV2 affect the biological function of the virus [45-49].

The 17th pair of primers and probes is used to be recommended by the US CDC, involving 110 isolates, the second most among 21 pairs of primers and probes. N: 28688 is the most common mutation sites among the above sequences, and at this site, the United States has the third most isolates since May $12^{\text {th }}, 2020$. And this pair of primers and probes was removed in the US CDC recommended protocol updated in July [50]. Whereas at other common mutation sites like N:28881- N:28883, ORF1ab:490, ORF1ab: 514, ORF1ab: 13402 and N: 28311, the region where mutant strains often appear does not overlap with the region where this kit is used (Figure S1). And in the selection and application of detection kits in the future, the 18th pair of primers and probes provided by China CDC would better not be used in the United Kingdom, Belgium, Netherlands and Portugal (Figure 3). Japan National Institute of Infectious Diseases recommended No.1 and No.3 pairs of primers and probes. The first pair should be avoided in the US and the third one should be avoided in Netherlands, the UK and the US (Figure 4A, 4B, S1A and S1B). It would be better not to use the 5th pair in 
Belgium (Figure 4C). Singapore, India and Australia would better avoid using the 15 th pair of primers and probes (Figure 4D and S1C).

There were some limitations to this study. Initially, the sequences of primers and probes used in the nucleic acid detection kits were provided by the WHO. However, not all kits used worldwide were available, some of which were not open access. Although the virus strain data is updated frequently, the strains we used merely presented the situation before our research ended. In addition, it has been reported that variations in primer binding sites could affect PCR efficiency [51, 52]. Thus, we are supposed to further validate the relationship between variations and PCR efficiency in the future. In addition, how SNPs influence virus detection and virus pathogenicity needs to be further investigated.

\section{Conclusions}

By integrating 21 pairs of primer target sequences in regions ORF1ab, S, E, and $\mathrm{N}$ from 6198 SARS-CoV-2 strains, the total length of primer and probe target sequences was 938 bp, with 131 (13.97\%) variant loci, involving 2415 (38.96\%) isolates. The E region had the highest priority in target sequence selection, with $\mathrm{N}$ region the least priority. SNP was the most frequent variation with $\mathrm{C}$ to $\mathrm{T}$ transitions involving the most variant loci. $\mathrm{G}$ to $\mathrm{A}$ transitions and $\mathrm{G}$ to $\mathrm{C}$ transversions were the most common with the highest isolate density. Genomic variations at the three mutation sites N: 28881, N: 28882, and N: 28883 were the most commonly detected variations, including 608 SARS-CoV-2 strains from 33 countries, especially in the UK, Portugal, and Belgium. Herein, we 
comprehensively analyzed the genomic variations on the target sequences of the nucleic acid amplification tests, offering evidence to the optimization of primer and probe target sequence selection, providing implications for the identification of infected patients via the tests.

\section{List of abbreviations}

SARS-CoV-2: severe acute respiratory syndrome coronavirus 2

2019nCoVR: the 2019 Novel Coronavirus Resource

COVID-19: the coronavirus disease 2019

RT-PCR: reverse transcription polymerase chain reaction

cDNA: complementary DNA

RdRP: RNA-dependent RNA polymerase

UTR: untranslated region

S: spike

E: envelope

M: membrane

$\mathrm{N}$ : nucleocapsid

ORF: open-reading frame 
China CDC: the Chinese Center for Disease Control and Prevention

IP France: Institute Pasteur, Paris, France

US CDC: Centers for Disease Control and Prevention, the United States

NIID Japan: National Institute of Infectious Diseases, Japan

HKU: Charité, Germany; School of Public Health, Hong Kong University

NIH Thailand: the National Institute of Health, Thailand

SNP: single nucleotide polymorphism

UK: the United Kingdom

Declarations

Ethics approval and consent to participate

Not applicable

Consent for publication

Not applicable

\section{Availability of data and materials}

The datasets generated and analysed during the current study are available in the 2019nCoVR, https://bigd.big.ac.cn/ncov. 


\section{Competing interests}

The authors declare that they have no competing interests

\section{Funding}

The study was supported by the National Science and Technology Major Sub-Project (2018ZX10301402-002), the Technical Innovation Special Project of Hubei Province(2018ACA138), the National Natural Science Foundation of China (81772787, 81873452, and 81974405), and the Fundamental Research Funds for the Central Universities (2019kfyXMBZ024).

\section{Authors' contributions}

CC and RY contributed equally to this work. They analyzed and interpreted the data, and wrote the original draft. QG and FY conceptualized and designed the study, and revised the manuscript. SZ and DL advised on the conception and design of the study. WG, RL, SW, YY and JC helped with the collection of resources. JL, YY, XJ, GC and NJ helped with editing assistance. All authors vouch for the respective data and analysis, revised, approved the final version, and agreed to publish the manuscript.

\section{Acknowledgments}

The authors thank all health-care workers and people involved in fighting against

COVID-19. We would like to acknowledge the platform 2019nCoVR provided by the Chinese Academy of Sciences. We thank the seven institutes for the disclosure of 
nucleic acid test kit contents. We appreciate the WHO providing SARS-CoV-2 relevant data.

\section{References}

1. WHO Director-General's opening remarks at the media briefing on COVID-19 - 11 March 2020 [https:/www.who.int/dg/speeches/detail/whodirector-general-s-opening-remarks-at-the-media-briefing-on-covid-19---11march-2020]

2. Hui DS, E IA, Madani TA, Ntoumi F, Kock R, Dar O, Ippolito G, McHugh TD, Memish ZA, Drosten C et al: The continuing 2019-nCoV epidemic threat of novel coronaviruses to global health - The latest 2019 novel coronavirus outbreak in Wuhan, China. International journal of infectious diseases : IJID : official publication of the International Society for Infectious Diseases 2020, 91:264-266.

3. Template:COVID-19

pandemic

data [https://en.wikipedia.org/wiki/Template:COVID-19_pandemic_data]

4. The species Severe acute respiratory syndrome-related coronavirus: classifying 2019-nCoV and naming it SARS-CoV-2. Nature microbiology 2020, 5(4):536-544.

5. Liu Y, Ning Z: Aerodynamic analysis of SARS-CoV-2 in two Wuhan hospitals. 2020 .

6. New Coronavirus Pneumonia Diagnosis and Treatment Guidelines (Trial Version

[http://www.nhc.gov.cn/yzygj/s7653p/202003/46c9294a7dfe4cef80dc7f5912e b1989.shtml]

7. Cheng MP, Papenburg J, Desjardins M, Kanjilal S, Quach C, Libman M, Dittrich S, Yansouni CP: Diagnostic Testing for Severe Acute Respiratory Syndrome-Related Coronavirus-2. Annals of Internal Medicine 2020.

8. Bordi L, Nicastri E, Scorzolini L, Di Caro A, Capobianchi MR, Castilletti C, Lalle E, On Behalf Of Inmi Covid-Study G, Collaborating C: Differential diagnosis of illness in patients under investigation for the novel coronavirus (SARS-CoV-2), Italy, February 2020. Euro Surveill 2020, 25(8):2000170.

9. Udugama B, Kadhiresan P, Kozlowski HN, Malekjahani A, Osborne M, Li VYC, 
Chen H, Mubareka S, Gubbay JB, Chan WCW: Diagnosing COVID-19: The Disease and Tools for Detection. ACS Nano 2020.

10. Guan WJ, Liang WH, Zhao Y, Liang HR, Chen ZS, Li YM, Liu XQ, Chen RC, Tang CL, Wang $\mathrm{T}$ et al: Comorbidity and its impact on 1590 patients with Covid-19 in China: A Nationwide Analysis. The European respiratory journal 2020 .

11. Guan W-j, Ni Z-y, Hu Y, Liang W-h, Ou C-q, He J-x, Liu L, Shan H, Lei C-1, Hui DSC et al: Clinical Characteristics of Coronavirus Disease 2019 in China. New England Journal of Medicine 2020.

12. Beeching NJ, Fletcher TE, Beadsworth MBJ: Covid-19: testing times. $B M J$ 2020, 369:m1403.

13. Al Johani S, Hajeer AH: MERS-CoV diagnosis: An update. Journal of infection and public health 2016, 9(3):216-219.

14. Zumla A, Hui DS, Perlman S: Middle East respiratory syndrome. Lancet (London, England) 2015, 386(9997):995-1007.

15. Hui DS, Wong PC, Wang C: SARS: clinical features and diagnosis. Respirology (Carlton, Vic) 2003, 8 Suppl(Suppl 1):S20-24.

16. Xie X, Zhong Z: Chest CT for Typical 2019-nCoV Pneumonia: Relationship to Negative RT-PCR Testing. 2020:200343.

17. Li Y, Yao L, Li J, Chen L, Song Y, Cai Z, Yang C: Stability issues of RT-PCR testing of SARS-CoV-2 for hospitalized patients clinically diagnosed with COVID-19. Journal of medical virology.

18. Wenling W, Yanli X, Ruqin G, Roujian L, Kai H, Guizhen W, Wenjie T: Detection of SARS-CoV-2 in Different Types of Clinical Specimens. JAMA 2020, 323(18):-.

19. Pan Y, Long L, Zhang D, Yan T, Cui S, Yang P, Wang Q, Ren S: Potential falsenegative nucleic acid testing results for Severe Acute Respiratory Syndrome Coronavirus 2 from thermal inactivation of samples with low viral loads. Clin Chem 2020.

20. Zou L, Ruan F, Huang M, Liang L, Huang H, Hong Z, Yu J, Kang M, Song Y, Xia J et al: SARS-CoV-2 Viral Load in Upper Respiratory Specimens of Infected Patients. N Engl J Med 2020, 382(12):1177-1179. 
21. Chu DKW, Pan Y, Cheng SMS, Hui KPY, Krishnan P, Liu Y, Ng DYM, Wan CKC, Yang P, Wang Q et al: Molecular Diagnosis of a Novel Coronavirus (2019-nCoV) Causing an Outbreak of Pneumonia. Clinical Chemistry 2020, 66(4):549-555.

22. Corman VB, T.; Brünink, S.; Zambon, M. : Diagnostic Detection of Wuhan Coronavirus 2019 by Real-Time RT-PCR. In: World Health Organization. Geneva; 2020.

23. Summary table of available protocols in this document [https://www.who.int/docs/defaultsource/coronaviruse/whoinhouseassays.pdf?sfvrsn=de3a76aa 2]

24. SARS-COV-2 DIAGNOSTIC PIPELINE [https:/www.finddx.org/covid19/pipeline/]

25. Freeman WM, Walker SJ, Vrana KE: Quantitative RT-PCR: Pitfalls and Potential. BioTechniques 1999, 26(1):112-125.

26. Dieffenbach CW, Lowe TM, Dveksler GS: General concepts for PCR primer design. PCR methods and applications 1993, 3(3):S30-37.

27. Gorbalenya AE, Baker SC, Baric RS, de Groot RJ, Drosten C, Gulyaeva AA, Haagmans BL, Lauber C, Leontovich AM, Neuman BW et al: The species Severe acute respiratory syndrome-related coronavirus: classifying 2019nCoV and naming it SARS-CoV-2. Nature microbiology 2020, 5(4):536-544.

28. Zhu N, Zhang D, Wang W, Li X, Yang B, Song J, Zhao X, Huang B, Shi W, Lu $\mathrm{R}$ et al: A Novel Coronavirus from Patients with Pneumonia in China, 2019. New England Journal of Medicine 2020, 382(8):727-733.

29. Bosch BJ, van der Zee R, de Haan CA, Rottier PJ: The coronavirus spike protein is a class $I$ virus fusion protein: structural and functional characterization of the fusion core complex. Journal of virology 2003, 77(16):8801-8811.

30. Wu C, Liu Y, Yang Y, Zhang P, Zhong W, Wang Y, Wang Q, Xu Y, Li M, Li X et al: Analysis of therapeutic targets for SARS-CoV-2 and discovery of potential drugs by computational methods. Acta pharmaceutica Sinica B 2020 .

31. Duffy S: Why are RNA virus mutation rates so damn high? PLoS biology 2018, 16(8):e3000003. 
32. Pachetti M, Marini B, Benedetti F, Giudici F, Mauro E, Storici P, Masciovecchio C, Angeletti S, Ciccozzi M, Gallo RC et al: Emerging SARS-CoV-2 mutation hot spots include a novel RNA-dependent-RNA polymerase variant. Journal of Translational Medicine 2020, 18(1):179.

33. Wang C, Liu Z, Chen Z, Huang X, Xu M, He T, Zhang Z: The establishment of reference sequence for SARS-CoV-2 and variation analysis. 2020.

34. Nalla AK, Casto AM, Huang M-LW, Perchetti GA, Sampoleo R, Shrestha L, Wei Y, Zhu H, Jerome KR, Greninger AL: Comparative Performance of SARS-CoV-2 Detection Assays Using Seven Different Primer-Probe Sets and One Assay Kit. Journal of clinical microbiology 2020, 58(6):e0055700520 .

35. Vogels CBF, Brito AF, Wyllie AL, Fauver JR, Ott IM, Kalinich CC, Petrone ME, Casanovas-Massana A, Catherine Muenker M, Moore AJ et al: Analytical sensitivity and efficiency comparisons of SARS-CoV-2 RT-qPCR primerprobe sets. Nature microbiology 2020.

36. Zhao WM, Song SH, Chen ML, Zou D, Ma LN, Ma YK, Li RJ, Hao LL, Li CP, Tian DM et al: The 2019 novel coronavirus resource. Yi chuan = Hereditas 2020, 42(2):212-221.

\section{2019 nCoVR [https://bigd.big.ac.cn/ncov]}

38. Corman VM, Landt O, Kaiser M, Molenkamp R, Meijer A, Chu DK, Bleicker T, Brünink S, Schneider J, Schmidt ML et al: Detection of 2019 novel coronavirus (2019-nCoV) by real-time RT-PCR. Euro Surveill 2020, 25(3):2000045.

39. Yin C: Genotyping coronavirus SARS-CoV-2: methods and implications. Genomics 2020.

40. Chang TJ, Yang DM, Wang ML, Liang KH, Tsai PH, Chiou SH, Lin TH, Wang CT: Genomic Analysis and Comparative Multiple Sequence of SARS-CoV2. Journal of the Chinese Medical Association : JCMA 2020.

41. Yao H, Lu X, Chen Q, Xu K, Chen Y, Cheng L, Liu F, Wu Z, Wu H, Jin C et al: Patient-derived mutations impact pathogenicity of SARS-CoV-2. medRxiv 2020:2020.2004.2014.20060160.

42. Suo T, Liu X, Feng J, Guo M, Hu W, Guo D, Ullah H, Yang Y, Zhang Q, Wang $X$ et al: ddPCR: a more accurate tool for SARS-CoV-2 detection in low viral load specimens. Emerging Microbes \& Infections 2020:1-30. 
43. Liu X, Feng J, Zhang Q, Guo D, Zhang L, Suo T, Hu W, Guo M, Wang X, Huang $Z$ et al: Analytical comparisons of SARS-COV-2 detection by qRT-PCR and ddPCR with multiple primer/probe sets. 2020:1-12.

44. Sapoval N, Mahmoud M, Jochum MD, Liu Y, Leo Elworth RA, Wang Q, Albin D, Ogilvie H, Lee MD, Villapol S et al: Hidden genomic diversity of SARSCoV-2: implications for qRT-PCR diagnostics and transmission. 2020.

45. Treangen TJ: bioRxiv.

46. Asselta R, Paraboschi EM, Mantovani A, Duga S: ACE2 and TMPRSS2 variants and expression as candidates to sex and country differences in COVID-19 severity in Italy. Aging 2020, 12(11):10087-10098.

47. Becerra-Flores M, Cardozo T: SARS-CoV-2 viral spike G614 mutation exhibits higher case fatality rate. 2020:e13525.

48. Pachetti M, Marini B, Benedetti F, Giudici F, Mauro E, Storici P, Masciovecchio C, Angeletti S, Ciccozzi M, Gallo RC et al: Emerging SARS-CoV-2 mutation hot spots include a novel RNA-dependent-RNA polymerase variant. $J$ Transl Med 2020, 18(1):179.

49. Chen J, Wang R, Wang M, Wei GW: Mutations Strengthened SARS-CoV-2 Infectivity. Journal of molecular biology 2020.

50. CDC's Diagnostic Test for COVID-19 Only and Supplies [https://www.cdc.gov/coronavirus/2019-ncov/lab/virus-requests.html]

51. Kalle E, Kubista M, Rensing C: Multi-template polymerase chain reaction. Biomolecular Detection and Quantification 2014, 2:11-29.

52. Kwok S, Kellogg DE, McKinney N, Spasic D, Goda L, Levenson C, Sninsky $\mathrm{JJ}$ : Effects of primer-template mismatches on the polymerase chain reaction: human immunodeficiency virus type 1 model studies. Nucleic acids research 1990, 18(4):999-1005.

Figure legends

Fig 1: Variation landscape of primer and probe target sequences in SARS-CoV-2 strains. Variant loci and numbers of mutant strains in ORF1ab (A), S (B), E (C), and $\mathrm{N}$ region (D). (E) Comparison of the number of variant loci in the four regions. (F) 
The number of variant loci (gray) and variation density (blue) in the four regions. (G) Mutant strains (light green) and strain density (green) of the four regions.

Fig 2: Variation analysis of SARS-CoV-2 strains in the target sequence of the nucleic acid amplification tests. (A) Pie chart of variation types. (B) Pie chart of SNP types. (C) Strain number and strain density of each SNP type. (D) Pie chart of the SNP types in ORF1ab, S, E, and N regions.

Fig 3: Variation analysis of N:28881, N: 28882, and N:28883 loci. (A) Variation landscape of primers F-18 and R-18. (B) Venn of the mutant strains containing variation in $\mathrm{N}: 28881, \mathrm{~N}: 28882$, or $\mathrm{N}: 28883$. (C) Variation curve of $\mathrm{N}: 28881, \mathrm{~N}$ : 28882, and N:28883 over time and by country. (D) The number of isolates and the variation frequency of $\mathrm{N}: 28881, \mathrm{~N}: 28882$, and $\mathrm{N}: 28883$ by country. Regional variances are shown at the top; blue histogram indicates isolate number; and the black line chart indicates variation frequency.

Fig 4: Isolates analysis of common variant loci. The number of isolates and the variation frequency of ORF1ab: 490 (A), ORF1ab: 514 (B), ORF1ab: 13402 (C), N: 28311 (D), and N: 28688 (E). Regional variances are shown at the top; blue histogram indicates isolate number; and the black line chart indicates variation.

Table 1. Polymerase Chain Reaction kits for SARS-CoV-2 and Variations

\begin{tabular}{cccccccc}
\hline No. & Region & & Sequence & Variation Sites & Variation & Isolates & Institute \\
\hline $\mathbf{1}$ & ORF1ab & F & TTCGGATGCTCGAACTGCACC & 3 & SNP & 37 & NIID Japan \\
& & R & CTTTACCAGCACGTGCTAGAAGG & 3 & SNP & 24 & \\
\hline $\mathbf{2}$ & ORF1ab & F & CTCGAACTGCACCTCATGG & 3 & Deletion, SNP & 5 & NIID Japan
\end{tabular}




\begin{tabular}{|c|c|c|c|c|c|c|c|}
\hline & & $\mathrm{R}$ & CAGAAGTTGTTATCGACATAGC & 5 & SNP & 17 & \\
\hline \multirow[t]{2}{*}{3} & ORF1ab & $\mathrm{F}$ & ACCTCATGGTCATGTTATGG & 7 & SNP, Deletion & 93 & NIID Japan \\
\hline & & $\mathrm{R}$ & GACATAGCGAGTGTATGCC & 1 & SNP & 1 & \\
\hline \multirow[t]{3}{*}{4} & ORF1ab & $\mathrm{F}$ & ATGAGCTTAGTCCTGTTG & 5 & SNP & 10 & NIID Japan \\
\hline & & $\mathrm{R}$ & CTCCCTTTGTTGTGTTGT & 0 & SNP & 0 & \\
\hline & & $\mathrm{P}$ & AGATGTCTTGTGCTGCCGGTA & 1 & SNP & 2 & \\
\hline \multirow[t]{3}{*}{5} & ORF1ab & $\mathrm{F}$ & CCCTGTGGGTTTTACACTTAA & 1 & SNP & 1 & China CDC \\
\hline & & $\mathrm{R}$ & ACGATTGTGCATCAGCTGA & 1 & SNP & 1 & \\
\hline & & $\mathrm{P}$ & CCGTCTGCGGTATGTGGAAAGGTTATGG & 3 & SNP & 53 & \\
\hline \multirow[t]{3}{*}{6} & ORF1ab & $\mathrm{F}$ & GGTAACTGGTATGATTTCG & 0 & SNP & 0 & IP France \\
\hline & & $\mathrm{R}$ & CTGGTCAAGGTTAATATAGG & 1 & SNP & 2 & \\
\hline & & $\mathrm{P}$ & TCATACAAACCACGCCAGG & 2 & SNP & 3 & \\
\hline \multirow[t]{4}{*}{7} & ORF1ab & $\mathrm{F}$ & GTGARATGGTCATGTGTGGCGG & 2 & SNP & 2 & Charité, Germany \\
\hline & & $\mathrm{R}$ & CARATGTTAAASACACTATTAGCATA & 0 & SNP & 0 & \\
\hline & & $\mathrm{P}$ & CCAGGTGGWACRTCATCMGGTGATGC & 2 & SNP & 3 & \\
\hline & & $\mathrm{P}$ & CAGGTGGAACCTCATCAGGAGATGC & 2 & SNP & 3 & \\
\hline \multirow[t]{3}{*}{8} & ORF1ab & $\mathrm{F}$ & GTGARATGGTCATGTGTGGCGG & 2 & SNP & 2 & Charité, Germany \\
\hline & & $\mathrm{R}$ & CARATGTTAAASACACTATTAGCATA & 0 & SNP & 0 & \\
\hline & & $\mathrm{P}$ & CAGGTGGAACCTCATCAGGAGATGC & 2 & SNP & 3 & \\
\hline \multirow[t]{3}{*}{9} & ORF1ab & $\mathrm{F}$ & TGGGGYTTTACRGGTAACCT & 1 & SNP & 11 & $\mathrm{HKU}$ \\
\hline & & $\mathrm{R}$ & AACRCGCTTAACAAAGCACTC & 4 & SNP & 8 & \\
\hline & & $\mathrm{P}$ & TAGTTGTGATGCWATCATGACTAG & 1 & SNP & 1 & \\
\hline \multirow[t]{2}{*}{10} & $\mathrm{~S}$ & $\mathrm{~F}$ & TTGGCAAAATTCAAGACTCACTTT & 5 & SNP & 19 & NIID Japan \\
\hline & & $\mathrm{R}$ & TGTGGTTCATAAAAATTCCTTTGTG & 1 & SNP & 1 & \\
\hline \multirow[t]{3}{*}{11} & S & $\mathrm{F}$ & TCAAGACTCACTTTCTTCCAC & 1 & SNP & 31 & NIID Japan \\
\hline & & & & 0 & & & \\
\hline & & $\mathrm{R}$ & ATTTGAAACAAAGACACCTTCAC & 1 & SNP & 2 & \\
\hline \multirow[t]{2}{*}{12} & $S$ & $\mathrm{~F}$ & AAGACTCACTTTCTTCCACAG & 9 & SNP & 30 & NIID Japan \\
\hline & & $\mathrm{R}$ & CAAAGACACCTTCACGAGG & 1 & SNP & 2 & \\
\hline \multirow[t]{3}{*}{13} & E & $\mathrm{F}$ & ACAGGTACGTTAATAGTTAATAGCGT & 1 & SNP & 1 & Charité, Germany \\
\hline & & $\mathrm{R}$ & ATATTGCAGCAGTACGCACACA & 1 & SNP & 1 & \\
\hline & & $\mathrm{P}$ & ACACTAGCCATCCTTACTGCGCTTCG & 3 & SNP & 6 & \\
\hline \multirow[t]{3}{*}{14} & E & $\mathrm{F}$ & ACAGGTACGTTAATAGTTAATAGCGT & 1 & SNP & 1 & IP France \\
\hline & & $\mathrm{R}$ & ATATTGCAGCAGTACGCACACA & 1 & SNP & 1 & \\
\hline & & $\mathrm{P}$ & ACACTAGCCATCCTTACTGCGCTTCG & 3 & SNP & 6 & \\
\hline \multirow[t]{3}{*}{15} & $\mathrm{~N}$ & $\mathrm{~F}$ & GACCCCAAAATCAGCGAAAT & 5 & SNP & 7 & US CDC \\
\hline & & $\mathrm{R}$ & TCTGGTTACTGCCAGTTGAATCTG & 3 & SNP & 25 & \\
\hline & & $\mathrm{P}$ & ACCCCGCATTACGTTTGGTGGACC & 3 & SNP & 42 & \\
\hline \multirow[t]{3}{*}{16} & $\mathrm{~N}$ & $\mathrm{~F}$ & CGTTTGGTGGACCCTCAGAT & 3 & SNP & 10 & NIH Thailand \\
\hline & & $\mathrm{R}$ & CCCCACTGCGTTCTCCATT & 6 & SNP & 8 & \\
\hline & & $\mathrm{P}$ & CAACTGGCAGTAACCA & 1 & SNP & 14 & \\
\hline \multirow[t]{3}{*}{17} & $\mathrm{~N}$ & $\mathrm{~F}$ & GGGAGCCTTGAATACACCAAAA & 2 & SNP & 90 & US CDC \\
\hline & & $\mathrm{R}$ & TGTAGCACGATTGCAGCATTG & 4 & SNP & 7 & \\
\hline & & $\mathrm{P}$ & AYCACATTGGCACCCGCAATCCTG & 8 & SNP & 13 & \\
\hline
\end{tabular}




\begin{tabular}{|c|c|c|c|c|c|c|c|}
\hline \multirow[t]{4}{*}{18} & $\mathrm{~N}$ & $\mathrm{~F}$ & GGGGAACTTCTCCTGCTAGAAT & 8 & SNP & 18 & China CDC \\
\hline & & & & & & 38 & \\
\hline & & $\mathrm{R}$ & CAGACATTTTGCTCTCAAGCTG & 7 & SNP & 9 & \\
\hline & & $\mathrm{P}$ & TTGCTGCTGCTTGACAGATT & 0 & SNP & 0 & \\
\hline \multirow[t]{3}{*}{19} & $\mathrm{~N}$ & $\mathrm{~F}$ & AAATTTTGGGGACCAGGAAC & 2 & SNP & 14 & NIID Japan \\
\hline & & $\mathrm{R}$ & TGGCAGCTGTGTAGGTCAAC & 3 & SNP & 3 & \\
\hline & & $\mathrm{P}$ & ATGTCGCGCATTGGCATGGA & 6 & SNP & 8 & \\
\hline \multirow[t]{3}{*}{20} & $\mathrm{~N}$ & $\mathrm{~F}$ & TAATCAGACAAGGAACTGATTA & 2 & SNP & 6 & $\mathrm{HKU}$ \\
\hline & & $\mathrm{R}$ & CGAAGGTGTGACTTCCATG & 7 & SNP & 20 & \\
\hline & & $\mathrm{P}$ & CAAATTGTGCAATTTGCGG & 3 & SNP & 5 & \\
\hline \multirow[t]{3}{*}{21} & $\mathrm{~N}$ & $\mathrm{~F}$ & TTACAAACATTGGCCGCAAA & 3 & SNP & 7 & US CDC \\
\hline & & $\mathrm{R}$ & GCGCGACATTCCGAAGAA & 0 & SNP & 0 & \\
\hline & & $\mathrm{P}$ & ACAATTTGCCCCCAGCGCTTCAG & 3 & SNP & 6 & \\
\hline
\end{tabular}

China CDC: the Chinese Center for Disease Control and Prevention; IP France: Institute Pasteur, Paris, France; US CDC: Centers

for Disease Control and Prevention, the United States; NIID Japan: National Institute of Infectious Diseases, Japan; HKU: School

of Public Health, Hong Kong University; NIH Thailand: the National Institute of Health, Thailand. 
Figures

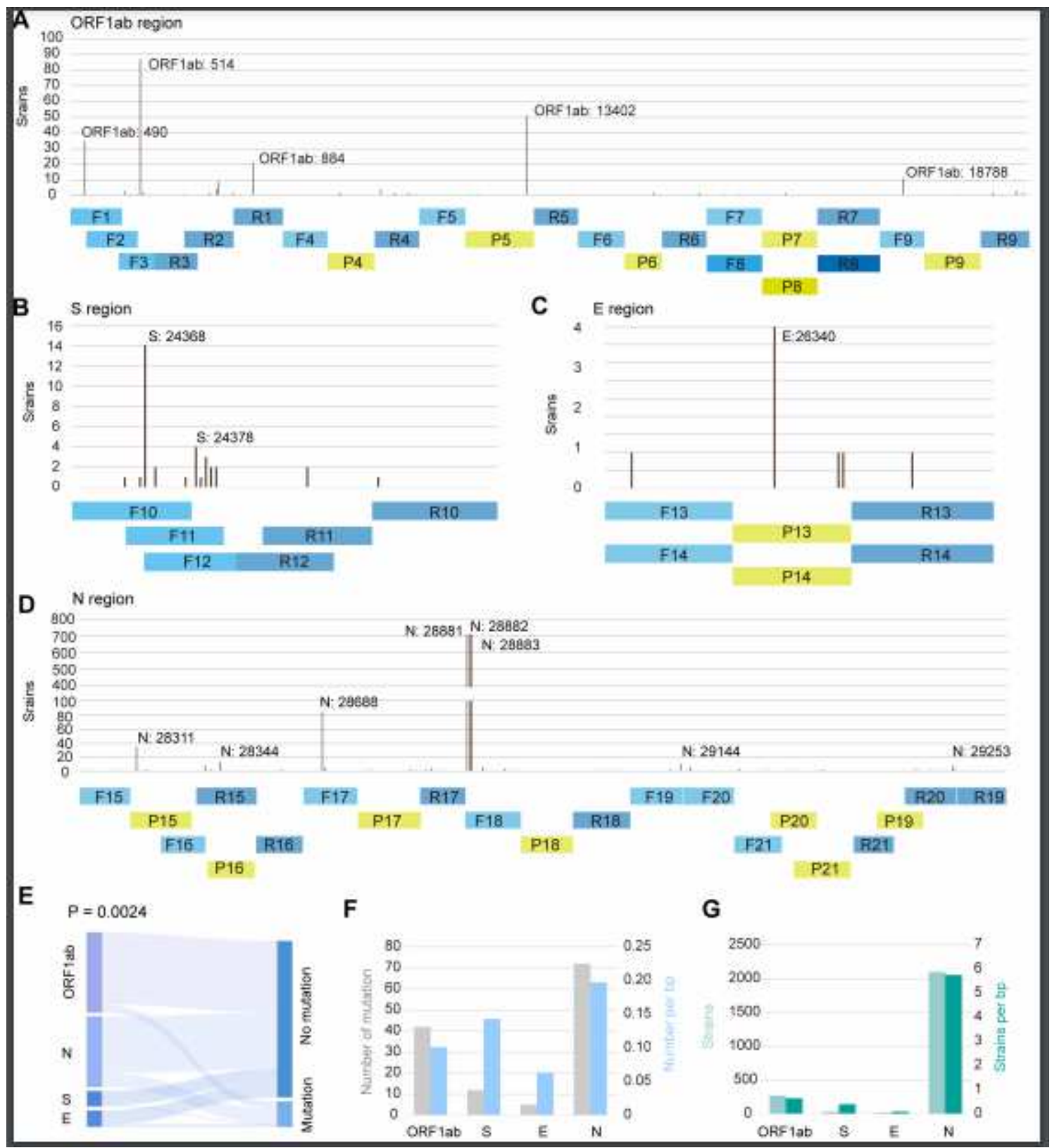

Figure 1

Variation landscape of primer and probe target sequences in SARS-CoV-2 strains. Variant loci and numbers of mutant strains in ORF1ab (A), S (B), $E(C)$, and $N$ region (D). (E) Comparison of the number of variant loci in the four regions. (F) 26 The number of variant loci (gray) and variation density (blue) in the four regions. (G) Mutant strains (light green) and strain density (green) of the four regions. 


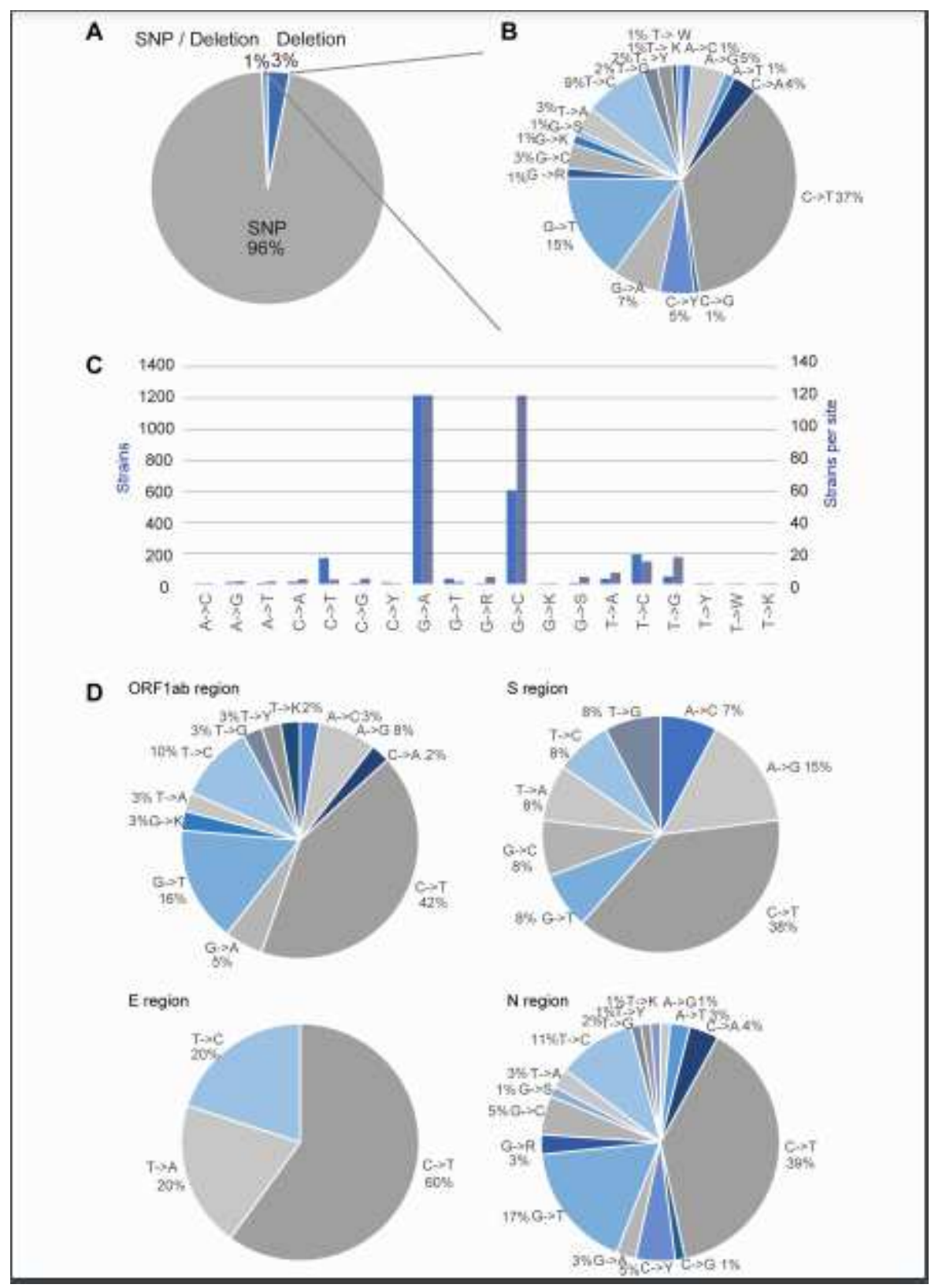

Figure 2

Variation analysis of SARS-CoV-2 strains in the target sequence of the nucleic acid amplification tests. (A) Pie chart of variation types. (B) Pie chart of SNP types. (C) Strain number and strain density of each SNP type. (D) Pie chart of the SNP types in ORF1ab, S, E, and N regions. 


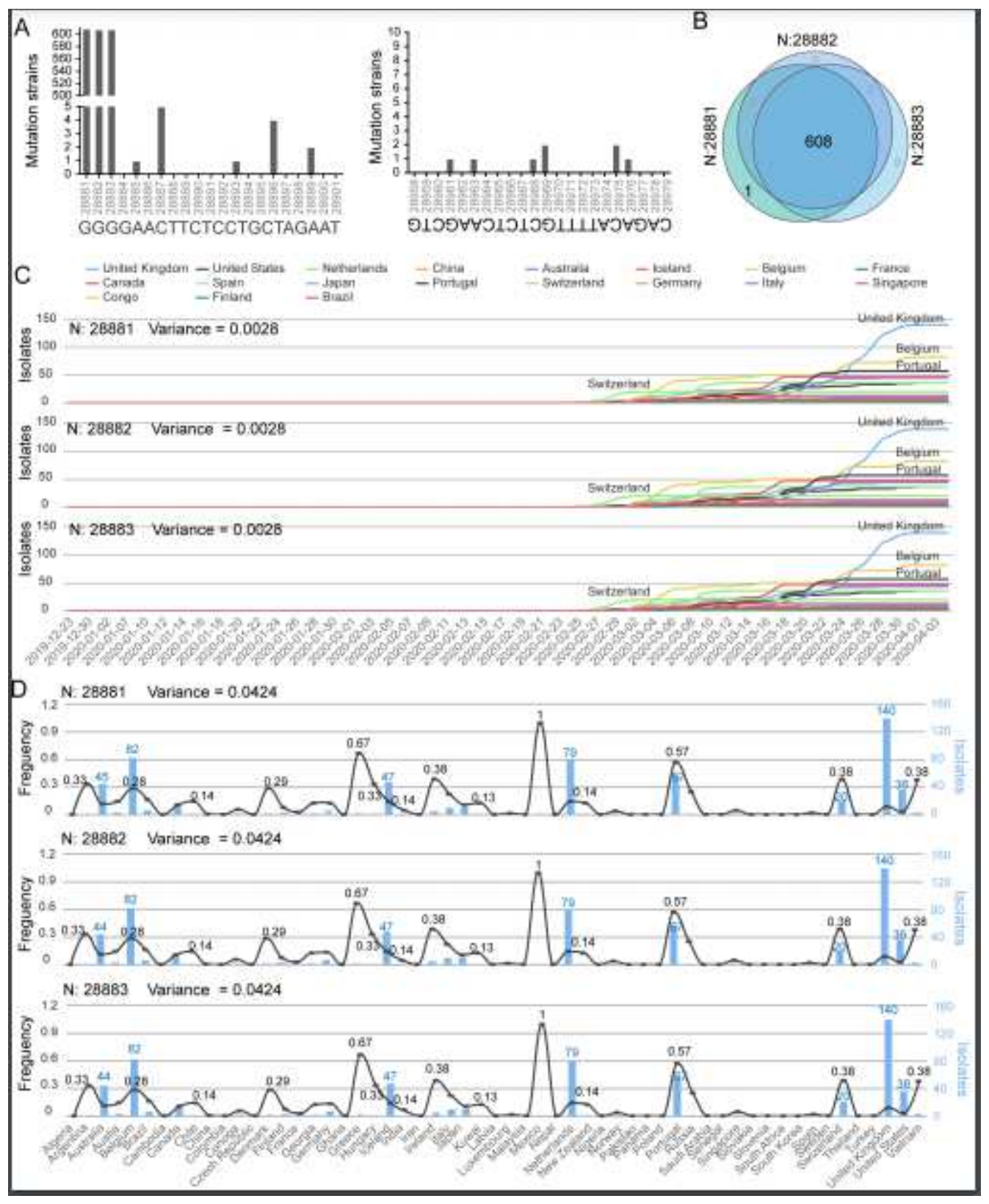

Figure 3

Variation analysis of N:28881, N: 28882, and N:28883 loci. (A) Variation landscape of primers F-18 and R18. (B) Venn of the mutant strains containing variation in $\mathrm{N}: 28881, \mathrm{~N}: 28882$, or $\mathrm{N}: 28883$. (C) Variation curve of N:28881, N: 28882 , and N:28883 over time and by country. (D) The number of isolates and the variation frequency of $\mathrm{N}: 28881, \mathrm{~N}: 28882$, and $\mathrm{N}: 28883$ by country. Regional variances are shown at the top; blue histogram indicates isolate number; and the black line chart indicates variation frequency. 


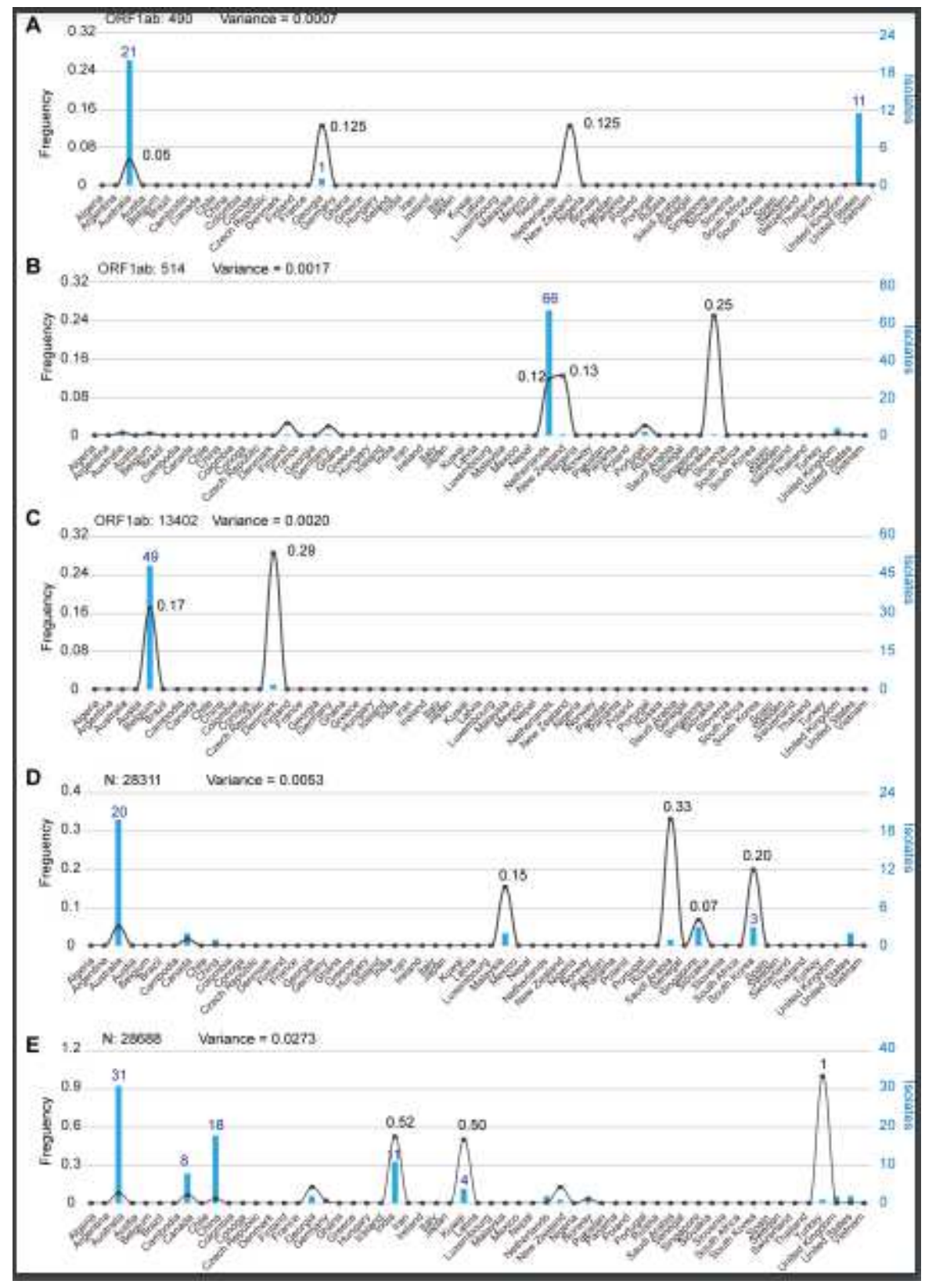

Figure 4

Isolates analysis of common variant loci. The number of isolates and the variation frequency of ORF1ab: 490 (A), ORF1ab: 514 (B), ORF1ab: 13402 (C), N: 28311 (D), and N: 28688 (E). Regional variances are shown at the top; blue histogram indicates isolate number; and the black line chart indicates variation.

\section{Supplementary Files}

This is a list of supplementary files associated with this preprint. Click to download.

- FigureS1.pdf

- S1Table.xIsx

- S2table.xIsx 
- S3tabel.xIsx

- S4Table.xIsx 